Die Entwicklungsgeschwindigkeit der gl-Zuchten gleicht der des Wildstammes. Die Vitalität wird durch gl nicht herabgesetzt. Das geht aus der Tatsache hervor, daß in allen Kreuzungsversuchen die Prozentzahl der Glasflügligen im allgemeinen nicht unter der Erwartung liegt.

Die neue gl-Mutante unterscheidet sich von der früheren glasflügligen Ephestia-Mutante durch folgende Eigenschaften: 1. Monofaktoriellen Erbgang. 2. Höhere Penetranz in den Reinzuchten $(99 \%$ statt $90 \%$ Merkmalsträger). 3. Die Heterozygoten der $\mathrm{F}_{1}$ zeigen vollkommene Dominanz der Normalbeschuppung über Glasflügligkeit (gegenüber fast vollständiger Dominanz, d. h. 0,5\% Entschuppte in $\mathrm{F}_{1}$ ).

\title{
Zur Frage eines Geschlechtsunterschiedes in den Nervenzellkernen von Drosophila
}

\author{
Von Thea LüERs \\ Aus dem Max-Planck-Institut für vergleichende Erbbiologie und Erbpathologie, Berlin-Dahlem \\ (Dir.: Prof. Dr. H. N a c h t s he i m) \\ (Z. Naturforschg. 10 b, 166-168 [1955]; eingegangen am 16. Dezember 1954) \\ Untersuchungen über die Größenverhältnisse des Nukleolar-Satelliten (Geschlechtschromatin) \\ in den großen Nervenzellen des Zentralnervensystems von Drosophila funebris und Droso- \\ phila melanogaster ergaben keine deutlichen Unterschiede zwischen Männchen und Weibchen.
}

$\mathrm{B}_{\mathrm{s}}$ arr und Bertram² beschrieben 1949 einen morphologischen Geschlechtsunterschied in den Nervenzellen $(\mathrm{Nz})$ erwachsener Katzen. Beim weiblichen Geschlecht fand sich in der Mehrzahl der Nz neben dem Nukleolus in den sonst optisch leeren Kernen ein Chromatinkörperchen von etwa $1 \mu$ Durchmesser, der Nukleolar-Satellit. Beim männlichen Geschlecht fehlte dieses Körperchen entweder oder es war nur spärlich ausgeprägt. Während der Nukleolus und die Niss l-Substanz bei der Niss l-Färbung violett erscheinen, färbt das Chromatinkörperchen sich blau. Bei der Feulgen-Methode verhält sich das Körperchen positiv, der Nukleolus negativ. Die Autoren waren in der Lage, nach Schnitten aus dem Gehirn, dem Rückenmark oder den sympathischen Ganglien der Katzen eine Gruppierung der Präparate nach dem Geschlecht vorzunehmen. Nur bei einer kleinen Anzahl von Individuen verhielt sich das Körperchen intermediär. In der Folge wurde von $\mathrm{B}$ a r r und Mitarbb. eine große Anzahl Tierarten nicht nur auf das Verhalten des Nukleolar-Satelliten in den Nervenzellen, sondern auch in anderen Körperzellen in der Interphase untersucht. Es zeigte sich, daß es sich um ein weit verbreitetes Phänomen handelt. Auch

1 Herrn Prof. Dr. W. S c h o l z zum 65. Geburtstag gewidmet.

2 M. L. B arr u. E. G. B e rtra m, Nature [London] 163, 676 [1949]. beim Menschen konnte in den Nervenzellen und in einer Reihe anderer Somazellen ein Geschlechtsunterschied festgestellt werden ${ }^{3}$. Davids on und Smith ${ }^{4}$ beschrieben sogar bei den segmentkernigen neutrophilen Leukozyten des Menschen einen Unterschied zwischen männlichen und weiblichen Individuen in Form eines kleinen, besonders gestalteten Segmentanhanges bei den Frauen (,drumstick“). Es zeigte sich aber auch, daß die Bezeichnung Nukleolar-Satellit zu eng gewählt war, da das Körperchen bei verschiedenen Arten und Geweben, beispielsweise in den Gliazellen, nicht neben dem Nukleolus, sondern an der Kernmembran liegend angetroffen wird. B arr und Mitarbb. sprechen daher heute von Geschlechts-Chromatin („sex chromatin“). Bei einigen Arten (Nager) konnte in den $\mathrm{Nz}$ kein Geschlechtsunterschied aufgefunden werden.

Nach diesen Mitteilungen schien es von Interesse, die Untersuchungen auf die imaginalen Nervenzellen von Drosophila auszudehnen. Aus früheren Arbeiten verfügen wir über etwa 500 in Paraffinserien geschnittene und nach $\mathrm{N}$ is s l gefärbte Exemplare von Drosophila melanogaster und Drosophila funebris. Ferner steht eine kleine Anzahl von Feulgen-

3 K. L. M o ore u. M. L. B a rr, J. comparat. Neurol. 98, 213 [1953]; Acta anat. 21, 197 [1954].

4 W. M. D a vids on u. D. R. S m it h, Brit. Med. Journ. 11, 6 [1954]. 
Präparaten zur Verfügung. Die Schnittdicke beträgt $5-7 \mu$.

Im Zentralnervensystem von Drosophila lassen sich bei der N i s s l - Färbung 3 Hauptgruppen von Nervenzellen unterscheiden: 1 . große plasma-reiche, 2 . kleine plasmaarme und 3. sehr kleine, bei denen ein basisch tingierbarer Plasmaleib im allgemeinen nicht erkennbar ist. Der erste Typ entspricht S pielmeyers ,somatochromen Zellen“, die ihren Schwerpunkt im Zelleib haben, der zweite Typ entspricht S pielmeyers „karyochromen Zellen“, die ihren Schwerpunkt im Zellkern haben, und der dritte Typ, der bei Drosophila nur im Lobus opticus des Kopfzentrums vorkommt, ist morphologisch mit den Körnerzellen des Kleinhirns der Säuger vergleichbar. Diese Zelltypen sind nach einem konstanten Muster bilateral symmetrisch im Zentralnervensystem angeordnet.

Bei dem großen Zelltyp erkennt man in den groBen hellen Zellkernen leicht den Nukleolus und ein ihm angelagertes Chromatinkörperchen, das sich bei der Feulgen-Methode positiv verhält. Für die Untersuchung dieses Chromatinkörperchens bei den beiden Geschlechtern wurden insgesamt 122 nach $\mathrm{N}$ is s 1 gefärbte Schnittserien herangezogen, und zwar 21 Exemplare der Art funebris und 101 Exemplare der Art melanogaster. 66 Individuen dieses Materials sind weiblich, 56 männlich. Auf einer Schnittserie sind je nach der Schnittführung etwa 3-20 Zellen des größten Zelltyps getroffen, die zur Untersuchung der Struktur des Zellkerns geeignet sind.

\section{Befunde und Diskussion}

Schon bei der ersten orientierenden Durchsicht zeigte sich, daß bei dem einzelnen Individuum die Größe der Nukleolar-Satelliten in verschiedenen Nervenzellen des somatochromen Typus Schwankungen unterliegt. Bei beiden Geschlechtern ist in manchen $\mathrm{Nz}$ überhaupt kein Satellit aufzufinden, in anderen ein spärlicher und schließlich in einigen ein recht großer. Manchmal projiziert sich der Satellit auf den Nukleolus, ist aber infolge seiner andersartigen Blaufärbung trotzdem erkennbar (Abb. $1^{*}-4$ ). Bei 7 Weibchen und 6 Männchen unter 101 Individuen von D. mel. war in keinem der auf den Schnitten getroffenen großen $\mathrm{Nz}$ ein Satellit nachweisbar. Bei 1 Männchen von $D$. fun. und bei 3 Weibchen von D. mel. wurde in jeweils einer Zelle ein DoppelSatellit von der Form eines Diplokokkus beobachtet (Abb. 5). Bei 1 Weibchen und 1 Männchen von D. mel. lag der Satellit dem Nukleolus nicht direkt an, sondern war durch einen feinen Faden mit diesem verbunden. Bei einigen Weibchen und Männchen bei-

* Abb. $1-6$ s. Tafel S. 168 a. der Arten befand sich in einzelnen $\mathrm{Nz}$ ein ausgesprochen fadenförmiger Satellit (Abb.6). Vereinzelt kommen 2 Satelliten vor, welche auf verschiedenen Seiten des Nukleolus diesem in derselben Schnittebene anlagern. $\mathrm{Ab}$ und zu trifft man in einem Kern außer dem Satelliten 1, manchmal auch 2 Chromatinkörperchen frei im Kernraum. Bei Beurteilung der Größenverhältnisse der Satelliten nach Betrachtung mit Okular 8 und $1 / 12$ Öl-Immersion konnte ein deutlicher Unterschied zwischen männlichen und weiblichen $\mathrm{Nz}$ nicht aufgefunden werden. Man hatte zwar manchmal den Eindruck, als sei der Satellit bei den Männchen etwas größer, aber der Versuch, die Präparate ohne Kenntnis des Geschlechtes zu ordnen, gelang nicht.

Barr u. a. nehmen in Übereinstimmung mit anderen Zytologen an, daß das von ihnen zunächst als Nukleolar-Satellit, später als Geschlechtschromatin bezeichnete feulgen-positive Körperchen aus den hetero-chromatischen Abschnitten der Geschlechtschromosomen stammt. Diese Abschnitte bleiben im Ruhekern kompakt. Bei Arten mit einem großen XChromosom und einem kleinen Y-Chromosom entsteht aus der Verschmelzung der hetero-chromatischen Abschnitte beider X-Chromosomen in weiblichen Körperzellen der große Satellit (,sex chromatin"), während in männlichen Geweben der XYChromosomenkomplex kein Körperchen von vergleichbarer Größe bildet, sofern das hetero-chromatische Y-Chromosom an Masse beträchtlich hinter dem hetero-chromatischen Anteil des $\mathrm{X}$ zurückbleibt. Bei den Vertretern der Rodentia, die ein großes Y-Chromosom besitzen, sind in beiden Geschlechtern wechselnde Zahlen von Chromatinkörpern im Kernraum sichtbar.

Eine Deutung der Befunde bei Drosophila erscheint auf Grund der Arbeiten von $\mathrm{Heitz}^{5}$ über somatische Heteropyknose und Geschlechtschromosomen bei verschiedenen Drosophila-Arten möglich. Wenn der Nukleolar-Satellit der Nz tatsächlich den hetero-pyknotischen Elementen der Geschlechtschromosomen entstammt, wie heute allgemein angenommen wird ${ }^{6}$, so dürfte ein wesentlicher Größenunterschied dieser Satelliten bei den beiden Geschlechtern bei Drosophila funebris und Drosophila melanogaster nicht zu erwarten sein. Drosophila funebris ist für solche Untersuchungen besonders geeignet, da nach

5 E. H e it z, Z. Zellforsch. mikroskop. Anatom. 19, 720 [1933]; 20, 237 [1933].

6 H. Hyden, Acta physiol. scand., 6 Suppl. 17 [1943]; C. u. O. V o g t, Ärztl. Forsch. 1, 8, 43 [1947]. 
$\mathrm{Heitz}$ ausschließlich die Geschlechtschromosomen ganz $(\mathrm{Y})$ oder partiell (X) hetero-chromatisch sind, während die Autosomen total euchromatisch sind. Bei etwa gleicher Länge beider Chromosomen ist das $\mathrm{X}$ mehr als zur Hälfte hetero-chromatisch; ein deutlich sichtbarer Unterschied zwischen der Masse des $\mathrm{XX}$-Komplexes und derjenigen des XY-Komplexes sollte demnach nicht zu erwarten sein. Bei Drosophila melanogaster liegen die Verhältnisse bezüglich der Geschlechtschromosomen ähnlich, jedoch enthalten bei dieser Art auch die beiden großen Auto- somenpaare im proximalen Bereich einen kurzen hetero-chromatischen Abschnitt. Nach B arr muß aber noch offen bleiben, wie weit noch nicht erfaßte unterschiedliche zellphysiologische Faktoren die bisher aufgedeckten Größenunterschiede des Geschlechtschromatins in den Körperzellen männlicher und weiblicher Individuen beeinflussen können. Zur weiteren Aufklärung dieser Fragen wurden Untersuchungen bei Drosophila-Ausnahmetieren mit abweichenden Zahlen von Geschlechtschromosomen (XO, XYY, XXY) begonnen.

\title{
Einfluß der Gehirnerschütterung auf den Übertritt von Methylenblau aus dem Blut in das Gehirn bei der Katze
}

\author{
Von G. Quadbeck und K. Randerath \\ Aus dem Max-Planck-Institut für Medizinische Forschung, Heidelberg, Institut für Chemie \\ (Z. Naturforschg. 10 b, 168-173 [1955]; eingegangen am 3. Dezember 1954)

\begin{abstract}
Schwere Gehirnerschütterungen verringern bei der Katze den Übergang von Methylenblau
\end{abstract} \\ aus dem Blut in das Hirngewebe. Durch Cocain wird diese Wirkung nicht beeinflußt. Die \\ Ursache des verringerten Farbstoffübertrittes ist nicht auf eine Abdichtung der Blut-Hirn- \\ Schranke, sondern wahrscheinlich auf eine trauma-bedingte, langanhaltende Stoffwechsel- \\ störung, welche die Reduktionsgeschwindigkeit von Methylenblau zu Leukomethylenblau \\ herabsetzt, zurückzuführen.
}

$\mathrm{D}$ er Übertritt einer Substanz aus dem Blut in das Zentralnervensystem (ZNS) wird von verschiedenen Faktoren beeinflußt. Neben sekretorischen Leistungen der Liquorproduktion sind vor allem Diffusionsvorgänge durch membranartige Schichten am Stoffaustausch wesentlich beteiligt. Die Geschwindigkeit des Stoffaustausches wird mit beeinflußt durch die Durchblutungsgröße im Bereich des ZNS. Weiterhin sind für manche Verbindungen auch stoffwechsel-gebundene reversible chemische Reaktionen, die nicht diffusible Stoffe so verändern, daß eine Diffusion möglich ist, anzunehmen. Alle diese verschiedenen Mechanismen können voneinander unabhängig wirken und sich hierbei gegenseitig verstärken, abschwächen oder in ihrer Wirkung sogar aufheben. Wenn im Folgenden von der Blut-ZNS-Schranke (BZS) die Rede ist, so verstehen wir hierunter allein die Steuerung des Übertrittes von im Blut gelösten Stoffes in das ZNS, wobei wir Durchblutungsgröße, sekretorische Leistungen der Liquorproduktion und alle Mechanismen, die einen an sich nicht „schrankenfähigen Stoff“ durch Änderung seiner chemischen Konstitution „schrankenfähig“ machen, bei der Beurteilung der Schrankenfunktion als solcher ausschließen wollen.

\footnotetext{
1 H. S p a t z, Arch. Psychiatr. 101, 267 [1934].

2 H. B e cker u. G. Quadbeck, Z. Naturforschg. 7b, 493 [1952].
}

Ein aus dem Blut in das ZNS übertretender Stoff läßt sich je nach seiner chemischen Konstitution im Liquor, in der Hirnsubstanz oder an beiden Orten nachweisen. Während z. B. viele Farbstoffe vorwiegend in der Hirnsubstanz fixiert werden, bleiben z. B. Ionen, soweit sie nicht in die Hirnsubstanz eingebaut werden, oft im Liquor frei gelöst. Diese Tatsache hat dazu geführt, daß man im wesentlichen auf Grund des unterschiedlichen Ortes für den Nachweis zwischen einer Blut-Hirn- und einer Blut-Liquor-Schranke differenzierte, und diesen beiden Schranken ein qualitativ unterschiedliches Verhalten zumaß 1 . Es besteht kein Zweifel, daß es im Bereich des ZNS Bezirke unterschiedlicher Schrankendurchlässigkeit gibt ${ }^{2}$. Ebenso kann auch nicht bestritten werden, daß z. B. beim postmortalen Zusammenbruch der BZS Triphenyl-tetrazolium-chlorid etwas früher im Liquor als in der Hirnsubstanz nachweisbar wird ${ }^{3}$. Trotzdem glauben wir, da bisher kein prinzipieller Unterschied zwischen der BHS und der Blut-Liquor-Schranke mit Sicherheit nachgewiesen werden konnte, mit dem Begriff BZS für beide Schranken zusammen auszukommen.

Der Einfluß geschlossener Schädeltraumen auf die Blut-ZNS-Schranke ist bereits mehrfach untersucht worden, ohne daß die Ergebnisse zu einem einheitlichen

3 H. Becker u. G. Quadbeck, Naturwissenschaften 37,265 [1950]. 